\title{
The epidemiology of rare heritable traits such as split-hand and foot malformation (SHFM)
}

\begin{abstract}
Deleterious traits with autosomal dominant mode of inheritance have low prevalence maintained by new mutations. Traditionally, estimates of mutation rate have been made by the assumed deterministic relation between prevalence and the balancing forces of selection and mutation. In this paper a simple model introducing stochastic elements is given. It illustrates how there can be substantial fluctuations in prevalence from generation to generation. The trait split-hand and foot malformation (SHFM) is used as an example of a condition to which the model might apply. Some features of the trait and its manifestation are sketched. The eminent British scientist Karl Pearson published some of the first systematic studies of a family with several individuals with SHFM over several generations. He examined the pattern of inheritance in the light of Mendel's model which was being strenuously debated at the time, concluding that SHFM did not conform well to Mendel's theory. There is now general acceptance that autosomal dominance is a valid model for some forms of SHFM. Pearson predicted that other species would provide useful models for the development of SHFM, as is amply demonstrated by ongoing studies of limb formation in Drosophila, chick and mouse.
\end{abstract}

Volume 10 Issue 2 - 2018

\author{
Alan Stark \\ Honorary Associate, School of Mathematics and Statistics, \\ University of Sydney, Australia
}

\author{
Correspondence: Alan Stark, Honorary Associate, School of \\ Mathematics and Statistics, FO7, University of Sydney, NSW \\ 2006, P.O. Box 479, Balgowlah, NSW, Australia, \\ Emailalans@exemail.com.au
}

Received: December 05, 2017 | Published: January 23, 2018

\section{Introduction}

The object of this paper is to describe a simple model of the occurrence of rare heritable traits. In the past, a standard deterministic model has been used to relate the incidence $A$ of a trait to the mutation rate $v$. One use of such a model is estimation of the mutation rate from the incidence. Here the emphasis is on the potential variability of occurrence by introduction of stochastic elements. The properties of the model are explored by simulation, assuming a set of values of the parameters.

An example of the use of incidence to estimate mutation rate is given by Penrose, in this case in relation to epiloia" "The absolute incidence of epiloia in the general population, which cannot be much greater than one in a hundred thousand, implies a mutation rate lower than this figure - a mutation frequency similar to that which would account for the persistence of haemophilia".

There are many traits, such as split-hand and foot malformation (SHFM), to which the model could be applied, but we use SHFM for illustrative purposes. Elliott et al. give the following summary. ${ }^{2}$

"SHFM is one of the most complex distal limb abnormalities. Highly variable in its presentation, SHFM can range from mild digital changes to a central cutaneous cleft without osseous deficiency to mild underdevelopment of the central digits (partial phalangeal type) to monodactyly (with only the fifth finger remaining). SHFM can occur as an isolated finding or as part of a complex syndrome. Over 75 such syndromes have been reported, and there is a significant phenotypic overlap among many of the syndromal forms. Approximately $40 \%$ of SHFM patients have associated non-limb congenital anomalies." They state that SHFM affects approximately one in 18,000 individuals, that is about 55 per million.

The traditional way of estimating the mutation rate of a dominant condition is by inverting the relation between the proportion of children born affected, the fitness and the mutation rate, leading to the estimate $\tilde{m}=\frac{1}{2} A(1-f)$, where $m$ is the mutation rate, $A$ is the incidence, and $f$ is the imputed ratio of the number of children born to affected individuals to the average family size in the whole population. This paper illustrates how $A$ can vary markedly by chance over a number of generations and so the estimate of $m$ will vary accordingly, even though the mutation rate is relatively constant. The model given in the third section makes provision for constructing $f$.

In the next section we give outlines of some studies which are typical of many in the literature. Section 3 contains the model of occurrence of the trait. Section 4 is a realization of the model from which various properties are revealed. Section 5 refers to contributions to research on SHFM by the eminent scientist Karl Pearson.

\section{Family trees of SHFM}

We summarize two papers which illustrate various features of SHFM. The first family was brought to the attention of H. Khosrovani by the surgeon who performed an operation to correct a malformation of the right hand of a girl who is shown on the pedigree to be one of 11 members of her generation (the fifth) to have malformation of the feet. ${ }^{3}$ Another of her generation is shown to have club foot. In all 25 members of the family were affected, of whom 19 were examined by Khosrovani and the remaining 6 were identified as affected by living descendants.

A member of the third generation, who is the parent or grandparent of many of those affected in the fourth and fifth generations, shows no anomalies in either hands or feet. Five out of eight of his sibs are affected. There are detailed descriptions of many of the members of the family and photographs and radiographs of various limbs.

Khosrovani gives the prevalence of SHFM as 1 in 112,000 , that is about 9 per million. He uses the terms ectrodactylie (ectrodactyly) and pince de homard (lobster-claw), the latter to give an impression of the external manifestation of the trait. He defines ectrodactyly as the congenital absence of one or more fingers and/or toes producing the lobster-claw appearance. He cites some of the early descriptions of the trait. He says the term Spalthand (split hand) was introduced in 1895. He notes that anomalies of the hands and feet are second only to those of the eye in humans with regard to frequency.

In his review of the literature, Khosrovani touches on many aspects of SHFM, such as different modes of inheritance, and 
variable expression and penetrance. He notes an early claim as to the hereditary nature of the trait which was made in 1858. In respect of the family which he studied, he concluded that it showed irregular autosomal dominant inheritance, since about equal number of males and females were affected and there were examples of male to male transmission. The family tree departs in some respects from the kind of model of transition from generation to generation introduced here. For example, there are cases where the parent of children with the trait is not affected. In these instances it is very difficult to escape the conclusion that the parent did carry the causative factor.

Khosrovani summarises the evidence of inheritance up to 1959. Many pedigrees of the kind presented by him suggest autosomal dominance, with about one third irregular, for example with apparent transmission by an unaffected individual.

As well as large family trees, families with small numbers of cases have been published. A chance observation sometime around 1922 by Dr. R. Fetscher led to the publication of a small pedigree of split hand. ${ }^{4}$ Fetscher's pedigree has two individuals with split hand. The index case is a boy aged twelve with both hands abnormal, but is otherwise normal. Three of his sibs have no observed abnormality. The remaining sib suffers from epilepsy. The father of the index case is the first cousin of the other affected individual in the family tree. This man has the same malformation as the index case. Fetscher claims that there is reason to believe that this family is an instance of recessive inheritance. However, this requires that the mother of the index case is a bearer of the same gene as the father, although the couple is not shown as consanguineous. The family tree illustrates the problem of genetic analysis if there is not DNA information. In recent years genome analysis has shown that several chromosomes have genes associated with the trait SHFM.

\section{The model}

We assume a cohort or population of fixed size $N$ which is renewed in discrete generations. A random number, which may be zero, of affected individuals, that is those with SHFM, is introduced in each generation. These arise by mutation from the class of unaffected individuals and so is the source of cases. New mutants come into the population at rate $v$ per chromosome and so is $2 v$ per person. A proportion $d$ of affected individuals carrying the mutation is not able to reproduce. Those who do produce copies of the mutation at rate $m$. A stochastic equilibrium of $A$ affected per generation is given by the relation

$$
A(1-d) m+2 N v=A
$$

leading to equilibrium mean

$$
A=\frac{2 N v}{1-m(1-d)}
$$

In the simulation reported here, the fertile carriers are selected by binomial sampling, as are the original mutants, and the numbers of copies of the mutation follow the Poisson distribution with parameter $m$. The number $A$ is a random variable and can be zero. Our aim is to explore its variation by simulation.

\section{A realization of the model}

We assume that the population has one million people, that is $N=$ $1,000,000$ and mutation rate $v=5 \times 10^{-7}$, so on average one mutant individual is formed per generation. The proportion 26/35 of affected individuals reproduce, that is $d=9 / 35$, and the rate at which fertile affected individuals reproduce children similarly affected is $m=5 / 4$.
Applying equation (2) gives mean $A=14$. As we note below, in the long run, the median number affected is 11 , which is about one fifth of the prevalence given by Elliott et al. ${ }^{4}$

In the simulation reported here, the population started with zero affected members and ran for 20,000 generations. A snapshot was taken of generations 9,801 to 10,200 . The numbers affected are displayed in Figure 1. The distribution of number affected per generation over all 20,000 generations is shown in Figure 2. The mean number is 14.12, standard deviation 11.56 and median number 11 . This is an indication of the stationary distribution, here fitted by a negative binomial distribution with parameters $\mathrm{k}=1.6190$ and $\mathrm{p}=0.1029$.

In Figure 2 the mean of the negative binomial distribution is $\tilde{k}(1-\tilde{p}) / \tilde{p}$ and the standard deviation $\sqrt{\tilde{k}(1-\tilde{p}) / \tilde{p}^{2}}$, producing values close to those given above. In the illustration used here, the balance between mutation and selection maintains the mutants arising over 14 generations, on average one per generation.

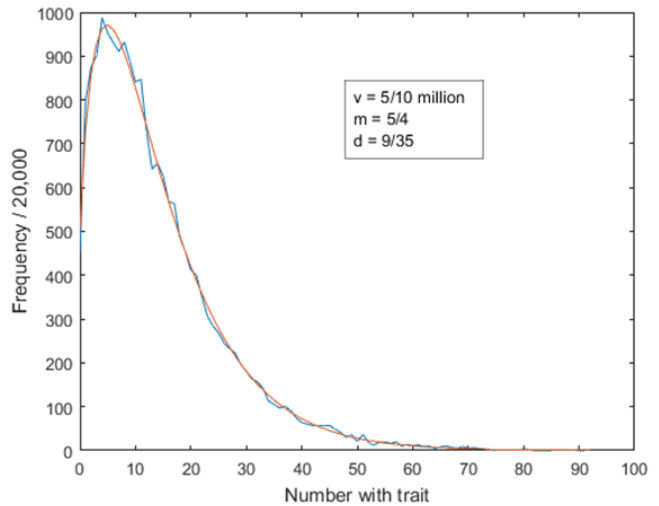

Figure I Empirical stationary distribution with fitted negative binomial distribution.

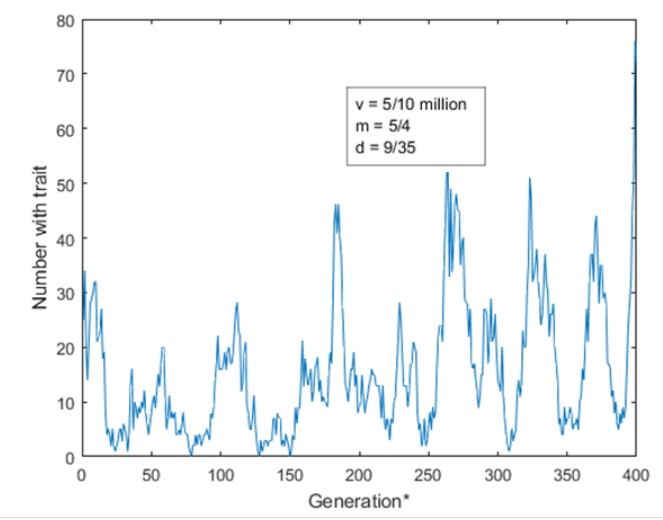

Figure 2 Time series of affected number over 400 generations, starting $9801 *$

In Figure 2 the simulation suggests that, from a cohort as large as one million, one may get a misleading estimate of the mutation rate, since in any generation the number affected may even be zero, or well above the rate given by equation (2). Of course, for larger populations, the sampling error will be lower. There is a high serial correlation between successive generations.

The model presented here is not Mendelian in that it considers only one parent of a child so that its main virtue may be that it is a simple way of giving insight to the occurrence of a trait. It is Mendelian in the sense that it is a particulate model of inheritance and perhaps more 
in keeping with Pearson's concept of inheritance as discussed in the next section.

\section{Contributions of karl pearson to research on SHFM}

Large pedigrees led to the notion that SHFM 'runs in families'. Lewis and Embleton published a remarkable study. ${ }^{5}$ They use the term 'sport', meaning mutation, "which takes place in the parental germ cells or their precursors". They believe that the family which they studied in great detail exhibited a distinct trait whose characteristics served to define the trait which 44 of them possessed. They describe in detail 17 of the 44 individuals of the family which they refer to as the "G" family. MacKenzie and Penrose traced the family investigated by Lewis and Embleton (1908) and ascertained many new cases which are displayed in their pedigree A. ${ }^{6}$

In the same year (1908) and in the same periodical Biometrika of which he was a founding editor, Karl Pearson described a family with SHFM. ${ }^{7}$ This was later revised with a co-author McMullan. ${ }^{8}$ Pearson made a detailed segregation analysis concluding that the data did not accord with Mendel's model of inheritance. This may have added to the widespread notion that Pearson did not accept the validity of Mendel's theory in the way that many scientists did and do. It may help to explain why Pearson's greatness is not as well recognized as it should be. In his account of "errors of the human body" Armand Marie Leroi includes a photograph of the hands of a child with SHFM together with the accompanying radiograph, taken from Pearson's paper of $1908 .{ }^{9}$

Stevenson and Jennings (1960) obtained information available in 1959 about the family tree initially investigated independently by Pearson and jointly by McMullan and Pearson. ${ }^{10}$ Although McMullan and Pearson did not accept the observed pattern of affected and non-affected family members as explicable by simple Mendelian inheritance, Stevenson and Jennings started from the hypothesis of autosomal dominant inheritance.

In 1932 Lancelot Hogben made a segregation analysis of five papers on SHFM which included those by Pearson and Lewis and Embleton. ${ }^{11}$ He gives a table whose heading is "Offspring of mating of affected and normal parents (split foot)". Hogben uses the counts of normal and affected children to make a statistical test of significance, concluding that autosomal dominant inheritance is an acceptable model, an inference contrary to that of Pearson and Lewis and Embleton. However, the situation is much more complex than this and SHFM is now divided into several categories, SHFM1, etc. OMIM states that 27 of 70 reported pedigrees of SHFM1 display regular autosomal dominant inheritance. ${ }^{12}$

Wang et al. ${ }^{13}$ describe a Chinese family with the form of SHFM classified as SHFM1. They give a simple pedigree consisting of three generations. The parents of the index case are shown as normal. The index case is a woman who has an affected son. Photographs of the woman show 'lobster-claw'-like feet and almost normal hands with slightly longer thumbs than normal. X-rays of the hands and feet reveal ectrodactyly and syndactyly of the feet and triphalangealy of the thumbs. The son of the index case has lobster-claw-like feet but no other visible abnormalities. Whole-exome sequencing of a DNA sample from the index case showed a missense mutation in exon 3 of $D L X 5$, and the same mutation was found in the son, but not in unaffected family members or controls. DLX5 has been mapped to chromosome $7 \mathrm{q} 22$. The authors give the mode of inheritance as autosomal dominant.
Pearson ${ }^{14}$ writes: "The existence of this digital deformity not only in the anthropoids, but also in the Old World monkeys, proves for it a long evolutionary history, and suggests that, had the mutation been of survival value, it might have been fixed as a specific character in one or other branch of the primates. It may someday be found possible, with increasing knowledge, to link species together by the occurrence of corresponding pathological states. ${ }^{14}$ ". The force of this statement is borne out remarkably in the case of SHFM. Several recent studies in Drosophila, chick, and mouse have revealed detailed information about the embryonic development of limbs ${ }^{15-18}$

The history of SHFM is like that of many conditions. It starts with observations of an apparent pattern of transmission from generation to generation, sometimes reported hundreds of years ago. In some disorders the bearers of the condition are noted in parish records. Soon after 1900, doctors and scientists began to view the patterns of occurrence in the light of Mendel's model of inheritance. Karl Pearson was one of the earliest to do this, not only in respect of SHFM but also in encouraging others to compile pedigrees systematically for a number of other disorders. The techniques of modern genetics are revealing ever-widening detail about numerous disorders, including SHFM. Pearson developed statistical tools for analysing data and models for interpreting evolution and this trend continues. The advance in knowledge leads to better advice and care for the community.

\section{Competing interest}

The author declares that he has no financial or other relevant conflict regarding this paper.

\section{Acknowledgements}

The author thanks Dr. W. Lanzing for translating the paper by Fetscher and a reviewer for suggesting ways to improve the manuscript.

\section{References}

1. LS Penrose. The Influence of Heredity on Disease. HK Lewis \& Co. Ltd, London, UK. 1934.

2. Elliott AM, Evans JA, Chudley AE. Split hand foot malformation (SHFM). Clinical Genetics. 2005;68(6):501-505.

3. Khosrovani H. Malformations des mains et des pieds (ectrodactylie) à travers cinq générations successives dans une grande famille Vaudoise. Journal de Génétique humaine. 1959;8:1-60.

4. Fetscher R. Ein Stammbaum mit Spalthand. Archiv für Rassen- und Gesellschafts-Biologie. 1922;14:176-177.

5. Lewis T, Embleton D. Split-hand and split-foot deformities, their types, origin, and transmission. Biometrika. 1908;6(1):26-58, 1908.

6. MacKenzie HJ, Penrose LS. Two pedigrees of ectrodactyly. Annals of Eugenics. 1951;16(1):88-96.

7. Pearson K. On inheritance of the deformity known as split-foot or lobster-claw. Biometrika. 1908;6(1):69-93.

8. McMullan G, Pearson K. On the inheritance of the deformity known as split-foot or lobster-claw. Biometrika. 1913;9(3-4):381-390.

9. Leroi AM. Mutants: On the Form. Varieties and Errors of the Human Body, Harper Perennial, London, New York, Toronto, Sydney. 2005.

10. Stevenson AC, Jennings LM. Ectrodactyly - evidence in favour of a disturbed segregation in the offspring of affected males. Annals of Human Genetics. 1960;24(1):89-96. 
11. Hogben L. Genetic Principles in Medicine and Social Science. Alfred A Knopf, New York, USA. 1932.

12. Online Mendelian Inheritance in Man (OMIM).

13. Wang X, Xin Q, Li L, et al. Exome sequencing reveals a heterozygous DLX5 mutation in a Chinese family with autosomal-dominant splithand/foot malformation. European Journal of Human Genetics. 2014;22(9):1105-1110.

14. Pearson K. On the existence of the digital deformity - so-called 'Lobster Claw' - in the apes. Annals of Eugenics. 1931;4:339-340.

15. Percival-Smith A, Ponce G, Pelling JJH. The noncell autonomous requirement of Proboscipedia for growth and differentiation of the distal maxillary palp during metamorphosis of Drosophila melanogaster. Genet Res Int. 2017;2624170.
16. Bastida MF, Ros MA. How do we get a perfect complement of digits ? Current Opinion in Genetics \& Development. 2008;18(4):374-380.

17. Tang WW, Kobayashi T, Irie N, et al. Specification and epigenetic programming of the human germ line. Nature Reviews Genetics. 2016;17(10):585-600.

18. Rattanasopha S, Tongkobpetch S, Srichomthong C, et al. Absent expression of the osteoblast-specific maternally imprinted genes, DLX5 and DLX6, causes split hand/split foot malformation type I. Journal of Medical Genetics. 2014;51(12):817-823. 